\title{
Survey on Acceptance of Passive Technology Monitoring for Early Detection of Cognitive Impairment
}

\author{
Sylvia Josephy-Hernandez ${ }^{a, b} \quad$ Catherine Norise ${ }^{a} \quad$ Jee-young Han ${ }^{a}$ \\ Kara M. Smith ${ }^{\text {a }}$ \\ aDepartment of Neurology, University of Massachusetts Medical School, Worcester, MA, USA; ${ }^{b}$ Behavioral \\ Neurology and Neuropsychiatry Fellowship Program, Massachusetts General Hospital, Boston, MA, USA
}

\section{Keywords}

Survey · Digital biomarkers · Cognition

\begin{abstract}
Introduction: Digital biomarkers may act as a tool for early detection of changes in cognition. It is important to understand public perception of technologies focused on monitoring cognition to better guide the design of these tools and inform patients appropriately about the associated risks and benefits. Health care systems may also play a role in the clinical, legal, and financial implications of such technologies. Objective: To evaluate public opinion on the use of passive technology for monitoring cognition. Methods: This was a one-time, Internet-based survey conducted in English and Spanish. Results: Within the English survey distributed in the USA $(n=173), 58.1 \%$ of respondents would be highly likely to agree to passive monitoring of cognition via a smartphone application. Thirty-eight percent of those with a higher degree of experience with technology were likely to agree to monitoring versus $20 \%$ of those with less experience with technology ( $p=0.003)$. Sixty-two percent of non-health-care professionals were likely to agree to monitoring versus $45 \%$ of health-care workers $(p=0.012)$. There were significant concerns regarding privacy $(p<0.01)$. We compared the sur-
\end{abstract}

karger@karger.com www.karger.com/dib

Karger"

GOPEN ACCESS
(C) 2020 The Author(s)

Published by S. Karger AG, Basel

This is an Open Access article licensed under the Creative Commons Attribution-NonCommercial-4.0 International License (CC BY-NC) (http://www.karger.com/Services/OpenAccessLicense), applicable to the online version of the article only. Usage and distribution for commercial purposes requires written permission. veys answered in Spanish in Costa Rica via logistic regression ( $n=43$, total $n=216$ ), adjusting for age, education level, health-care profession, owning a smartphone, experience with technology, and perception of cognitive decline. Costa Rican/Spanish-speaking respondents were 7 times more likely to select a high probability of agreeing to such a technology $(p<0.01)$. English-speaking respondents from the USA were 5 times more likely to be concerned about the impact on health insurance $(p=0.001)$ and life insurance $(p=$ $0.01)$. Conclusions: Understanding public perception and ethical implications should guide the design of digital biomarkers for cognition. Privacy and the health-care system in which the participants take part are 2 major factors to be considered. It is the responsibility of researchers to convey the ethical and legal implications of cognition monitoring.

$$
\begin{aligned}
& \text { (c) } 2020 \text { The Author(s) } \\
& \text { Published by S. Karger AG, Basel }
\end{aligned}
$$

\section{Introduction}

Due to a growing and ageing population, the global burden of dementia more than doubled from 1990 to 2016, to affect 43.8 million people [1]. Current tools for detecting and monitoring dementia are fraught with lim- 
itations. Traditional biomarkers including imaging assessment (e.g., PET) as well as biological evaluations (e.g., of the cerebrospinal fluid) have been utilized [2-4]. These traditional biomarkers, coupled with clinical and neuropsychological testing, have influenced the paradigm of assessment of dementia and mild cognitive impairment (MCI). However, these methods are invasive, time-consuming, expensive, and impossible to access for large portions of the world's population. Furthermore, better therapies to treat dementia are greatly needed, and new easyto-use tools have the potential to fuel the development of novel therapeutic options [5].

In an attempt to augment the clinical assessment and address the identified limitations, digital biomarkers have been explored. Digital biomarkers refer to the use of digital technologies (i.e., environmental sensors, portable, wearable, and/or implantable devices) to allow for the collection of physiological and behavioral data that is objective and quantifiable [6]. Digital biomarkers can be either active or passive. Active refers to where a participant directly interacts with the tool; passive refers to the tool collecting information about the participant without their direct engagement. In the case of memory disorders, the objective of such technologies is to capture subtle transitions in cognition. Digital biomarkers may augment imaging and biochemical biomarkers to facilitate the detection, prediction, and monitoring of memory disorders.

Several approaches to remote cognitive monitoring through use of technology have been shown to be feasible. Studies utilizing nonintrusive, at-home technologies like computer usage have shown promise as a viable metric for the early signs of cognitive decline [7]. Dodge et al. [8] demonstrated that in-home remote assessment of walking speed variability may be used as an early marker of MCI. A study by Austin et al. [9] analyzed how Internet searches relate to language and cognitive function in older adults. This study demonstrated that it may be possible to detect early signs of cognitive decline through Internet search terms. The current limitations of digital biomarkers are the lack of standardization of tools and established regulatory guidelines on privacy [10]. Importantly, there is a lack of understanding of the public's attitudes and acceptance to utilize these technologies, which we aim to address here. Though the term "digital biomarkers" includes a broad range of modalities, we focused this study on the passive monitoring of writing features. Such features may be seen as a manifestation of cognition [11], and this tool is a concrete measure that could be proposed to a lay-person audience.
There are also ethical issues surrounding the early diagnosis of memory disorders that must be explored. Since there are currently only limited treatments for dementia, one may question the benefit of screening, especially among asymptomatic individuals [12]. Authors in the past have discussed the "right to know and the wish not to know." An early diagnosis may lead to distress; however, patients do have the right to know their diagnosis and this may also allow for planning. A better understanding of the early stages of cognitive decline could not only allow for the modification of risk factors but could, itself, guide new therapeutic approaches. As with any test, patients who undergo cognitive screening should be informed of the potential implications prior to the evaluation [13].

Finally, attitudes and opinions towards screening may depend on the context of government, health care, and health insurance for an individual. We therefore compared opinions between participants at 2 different geographical locations and with different cultures and healthcare systems, namely, in the USA and Costa Rica.

\section{Materials and Methods}

Study data were collected and managed using REDCap electronic data capture tools hosted at the University of Massachusetts Medical School, Worcester, MA, USA $[14,15]$. Power calculations were limited due to the novelty of our study. Retrospectively, based on our survey sample size of $n=173$, a 2 -sided $95 \%$ CI for a single proportion would extend $0.425-0.575$ around 0.5 , providing us with confidence in the level of precision our survey provides.

The study consisted of a one-time, Internet-based survey conducted from January to April 2019. The survey was created using principles from a health survey design textbook [16]. Readability was assessed with a free online checker aiming for a 6th-grade reading level.

Surveys in English were distributed via e-mail through the UMass Conquering Diseases program to the US cohort. Surveys were distributed to participants who had registered as volunteers with interest in any of the following categories: all research studies, aging, Alzheimer's, behavioral health, healthy volunteers, and mental health. The survey in English was distributed to approximately 950 participants and was answered by 173 (18\% response rate). Surveys in Spanish were distributed via Facebook social media in a Costa Rican social circle as well as a Facebook group of Costa Rican physicians. It was therefore difficult to quantify the exact number of participants who had access to the survey.

The surveys can be accessed in the online supplementary material (see www.karger.com/doi/10.1159/000512207). They consisted of 21 questions: 11 demographic, 9 opinion (5-point Likert scale), and 1 open-ended for comments. The focus of the surveys was digital biomarkers, with a concrete example of passive monitoring of writing features. The only inclusion criterion was con- 
Table 1. Subject demographics and statistical comparisons between respondents to the English and Spanish surveys

\begin{tabular}{|c|c|c|c|c|}
\hline & $\begin{array}{l}\text { English } \\
(n=173)\end{array}$ & $\begin{array}{l}\text { Spanish } \\
(n=43)\end{array}$ & Test & $p$ value \\
\hline Age & & & Student $t$ & 0.002 \\
\hline Mean, years & 55.68 & 34.93 & & \\
\hline $\mathrm{SD}$, years & 15.778 & 11.569 & & \\
\hline Sex & & & $x^{2}$ & 0.847 \\
\hline Male & $33 \%$ & $34 \%$ & & \\
\hline Female & $67 \%$ & $66 \%$ & & \\
\hline Level of education & & & $x^{2}$ & 0.03 \\
\hline High school or below & $22 \%$ & $0 \%$ & & \\
\hline College & $78 \%$ & $100 \%$ & & \\
\hline Profession & & & $x^{2}$ & $<0.01$ \\
\hline Not in health care & $74 \%$ & $39 \%$ & & \\
\hline Health care & $26 \%$ & $61 \%$ & & \\
\hline Diagnosis of cognitive impairment & & & $x^{2}$ & 0.584 \\
\hline No & $97 \%$ & $95 \%$ & & \\
\hline Yes & $3 \%$ & $5 \%$ & & \\
\hline Relative with a diagnosis of cognitive impairment & & & $x^{2}$ & 0.598 \\
\hline No & $49 \%$ & $57 \%$ & & \\
\hline Yes & $51 \%$ & $43 \%$ & & \\
\hline Owns a smartphone & & & $x^{2}$ & 0.03 \\
\hline No & $10 \%$ & $0 \%$ & & \\
\hline Yes & $90 \%$ & $100 \%$ & & \\
\hline Level of knowledge of technology & & & $x^{2}$ & 0.112 \\
\hline Low & $7 \%$ & $7 \%$ & & \\
\hline Moderate to high & $93 \%$ & $93 \%$ & & \\
\hline Memory now versus 10 years ago & & & $x^{2}$ & 0.025 \\
\hline Poor & $19 \%$ & $5 \%$ & & \\
\hline Moderate to good & $81 \%$ & $95 \%$ & & \\
\hline
\end{tabular}

senting to the survey; exclusion criteria included not consenting to the survey or being under the age of 21 years. Only surveys with at least 1 complete opinion section were included in the statistical analysis.

The likelihood of agreeing to passive monitoring of cognition focused on the following question (on a 5-point Likert scale): "There is currently is no cure or effective treatment for dementia. Tools such as behavior monitoring could diagnose diseases earlier in time. Knowing the above, would you be willing to have your behavior monitored on your smart phone? (scale of $1-5,5$ being that you completely agree)."

Statistical analysis was performed using SPSS v21. Nonparametric statistics were used for data analysis. For comparing nominal values in 2 subpopulations, the $\chi^{2}$ test was used. For ordinal values, the Mann-Whitney U test was used to compare 2 subpopulations and Kruskal-Wallis test to compare $\geq 3$ subpopulations. Kendall's $W$ coefficient was used for interrater agreement regarding participants' concerns. A $p$ value $<0.05$ was considered statistically significant.

Demographic data were compared between surveys collected in English and Spanish using either a 2-tailed $t$ test or the $\chi^{2}$ test. If the $p$ value was $<0.2$, such demographic characteristics were included in a binary logistic regression analysis. The Likert scale was divided into "high" values (4 and 5), and "low" values (1-3). A p value $<0.05$ was considered statistically significant.

NVivo software (release 1.2) was used to analyze open-ended comments.

\section{Results}

Demographic data of participants in English and Spanish differed significantly in age, education level, health-care profession, owning a smartphone, and perception of cognitive decline (Table 1 ).

For the survey in English, 58.1\% of the participants replied they would be highly likely to agree to passive monitoring of cognition via a smartphone application (4 or 5 out of 5 on the Likert scale). Participants reporting a higher degree of experience with technology were more likely to agree to monitoring (Fig. 1, Kruskal-Wallis test $p=$ 0.003). Health-care professionals were less likely to agree to monitoring (Fig. 2, Mann-Whitney U test $p=0.012$ ). 


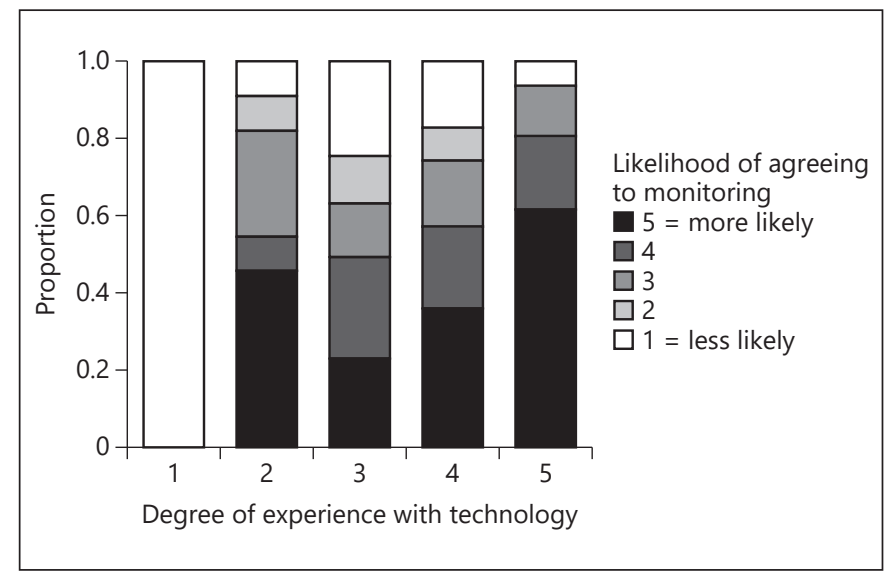

Fig. 1. A higher degree of experience with technology coincides with a higher likelihood of agreeing to monitoring. Comparison between the degree of experience with technology on a 5-point Likert scale ( $x$ axis) and the likelihood of agreeing to monitoring again on a 5-point Likert scale (color coded). Kruskal-Wallis test, $p=0.003$.

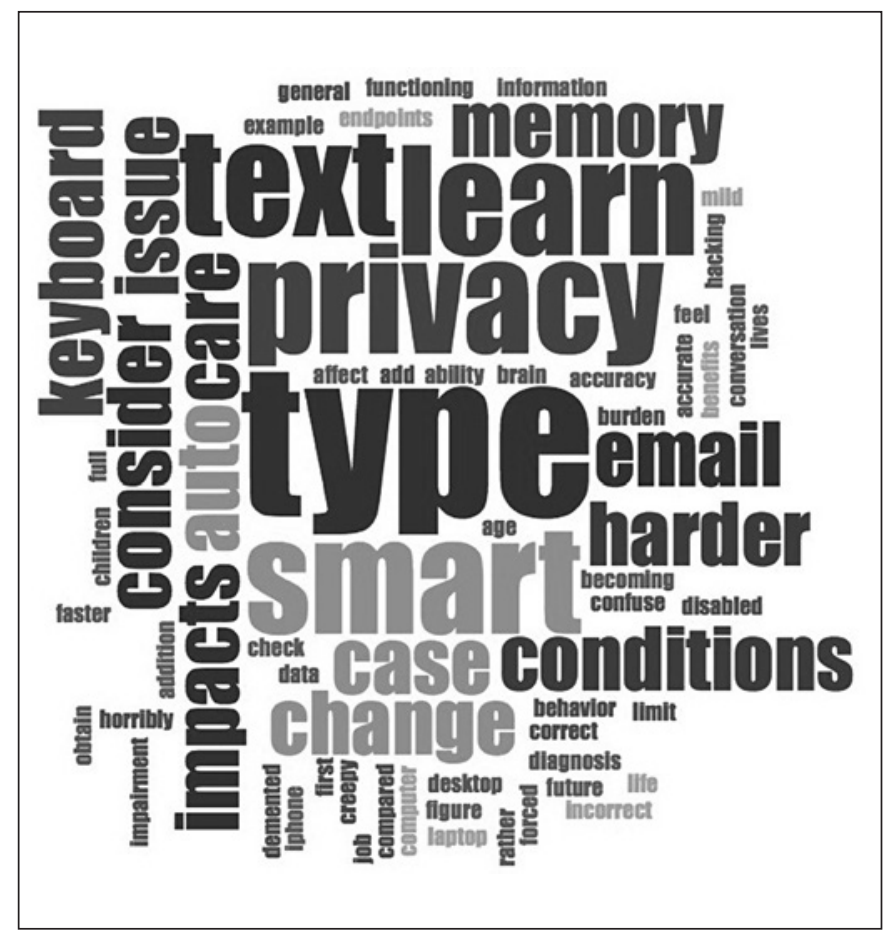

Fig. 3. Predominant topics within the open-ended comments were typing, privacy, and the ownership or use of a smartphone. NVivo software was used to create a word cloud in which font size was proportional to the frequency of a term within the open-ended comments.

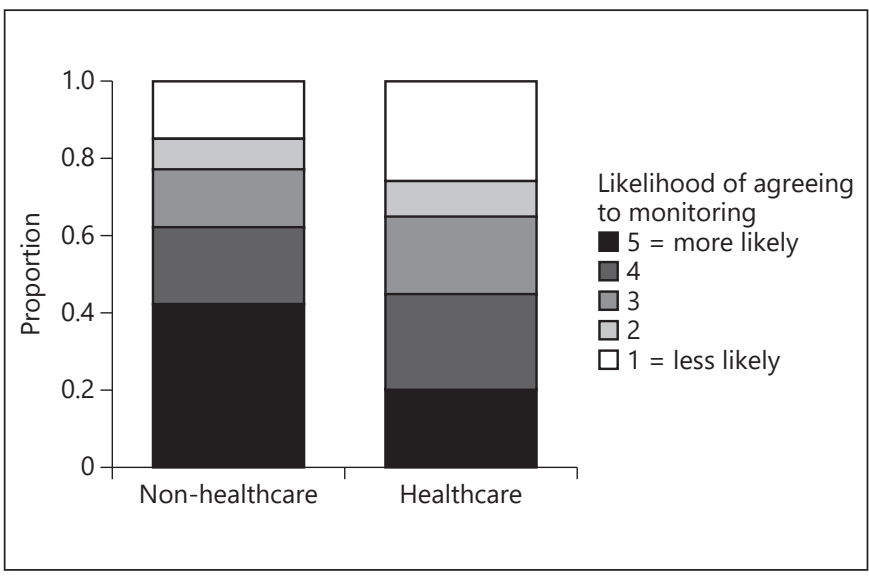

Fig. 2. Health-care professionals were less likely to agree to monitoring. Whether a participant was a health-care professional or not is displayed on the $x$ axis, and the likelihood of agreeing to monitoring on a 5-point Likert scale (color-coded). Mann-Whitney U test, $p=0.012$.

Sex, age, education level, a diagnosis of dementia in the respondent or a relative, and a self-perception of decline in cognition did not affect the participant's likelihood of agreeing to monitoring.

For the survey in Spanish, $88.4 \%$ of the participants replied they would be highly likely to agree to passive monitoring of cognition via a smartphone application (4 or 5 out of 5 on the Likert scale). Given the small number of responses in Spanish, we focused on a comparison to the survey answered in English. Via logistic regression $(n=43$, total $n=216$ ), we adjusted for age, education level, healthcare profession, owning a smartphone, knowledge of technology, and perception of cognitive decline. Respondents in Spanish were 7 times more likely to select a high probability of agreeing to such a technology $(p<0.01)$.

Respondents in both English and Spanish had privacy as their main concern (Kendall's $W, p<0.01$ ). However, respondents in English were next-most concerned about health insurance whereas respondents in Spanish were next-most concerned about anxiety regarding a diagnosis. Regression analysis showed that respondents in English were 5 times more likely to be concerned about the impact on health insurance $(p=0.001)$ and life insurance $(p=0.01)$.

There were 22 open-ended comments in English and 1 in Spanish. Comments were analyzed using the NVivo software. The most common topics were concerns regarding privacy, the accuracy of typing as a measure of cognition, and the lack of ownership or use of a smartphone (Fig. 3). Representative comments include: "Pri- 
vacy issues specifically hacking," "too many factors can affect the typing of text messages, including auto correct, and so on," "I barely ever use my smart phone for messaging. I use it as a phone, rarely use it unless necessary. I rather use email." The only comment in Spanish was regarding the possibility of a false-positive.

\section{Discussion}

Technology should be an essential tool in the discovery of treatments [17]. The increasing prevalence of dementia in our aging population makes cognition a logical target for the development of digital biomarkers. These tools may be valuable not only for tracking disease progression, but also for early diagnosis of cognitive illnesses. However, researchers must be transparent with the population about the implications of technology and the early knowledge of a diagnosis. We have provided this article as a tool to promote a design of passive screening and monitoring tools aligned with the needs of the population.

To our knowledge, this is the first article reporting the public's perception about digital passive screening of changes in writing as a measure of cognition and its potential outcomes. We showed moderate acceptability with the survey answered in English and high acceptability with the survey answered in Spanish (although it was difficult to generalize, given the small sample size of the latter). We focused on the use of technology and not the general question of screening of cognition, which has been previously addressed and discouraged by different authors [18]. We would, however, argue that an early diagnosis could lead to the discovery of underlying pathologies and modifiable risk factors as well as help to fuel the discovery of therapies to prevent disease progression. Future studies are needed to assess if our findings can be extrapolated to other types of digital biomarkers, including the passive monitoring of physiologic measures, spoken language, and mobility, among others.

Coravos et al. [19] published an excellent primer, setting the ethical stage for the development of digital biomarkers. They highlighted the necessity of obtaining informed consent that ensures that participants understand the technology and its implications. In our study, healthcare professionals were significantly less likely to agree to monitoring, possibly due to their better understanding of the implications of a diagnosis of cognitive impairment. Participants with greater experience with technology were more amenable to monitoring. This may suggest that, as the generation born with computers become old-

Acceptance of Passive Digital Biomarkers er, it will likely be more comfortable with specific types of monitoring. Lastly, informed consent needs to be tailored to the technological literacy of a participant.

In our study, the main concern of participants was privacy. This has been previously discussed in the context of digital markers for psychiatric illness $[20,21]$ as well as several neuropsychiatric conditions [10]. Applications and devices that monitor behavior and physiology may be designed so that the device itself processes the information, with no transmission of sensitive data. Such a design would help protect the privacy of the individual and enhance the acceptability of digital biomarkers. This is an important aspect to include in future surveys.

Despite adjusting for demographic factors, participants who answered the survey in Spanish in Costa Rica were more likely to participate in monitoring and less likely to be concerned with health insurance and life insurance than the respondents in the USA (a country with mainly private insurance companies and a lack of universal health care). The presence of universal health care in Costa Rica may have allowed for more concerns about the actual diagnosis than about the implications for insurance issues. The legal and financial implications of an early diagnosis should therefore be explored in each country where these technologies are to be implemented. For instance, in countries like the USA, where health insurance is often provided by employers, whether biomarker information would be made available to employers, health insurance companies, or life insurance companies would potentially influence public opinion.

The main limitations of our study include the small sample size, sample bias given it was an Internet-based survey asking questions about technology, and the fact that we could not be completely certain of a person's geographical location at the time of answering the survey. The relatively narrow distribution tool utilized may also limit the generalizability of the findings. Greater distribution efforts need to be undertaken to have a larger international sample. The fact that the English and Spanish surveys were distributed differently was partially due to the different preferences regarding communication in these 2 cultures. However, this difference may, at least partially, explain the demographic differences between the 2 cohorts. Despite these limitations, this study provides an initial exploratory investigation into public awareness and perception of the development of digital biomarkers.

Our survey may serve as a template for future studies exploring the public's acceptance of passive monitoring of cognition, language, and behavior. We hope to further explore opinions and obtain larger samples of partici- 
pants from different sociocultural backgrounds. It is also important to distinguish between cultures within the same geographical area and between geographical areas with different health-care systems. As mentioned above, it is important to include features that lessen privacy concerns and investigate whether our findings can be extrapolated to other technologies. Further data gathering should influence the design of monitoring tools and may also impact advocacy efforts to allow individuals to benefit from, rather than be harmed by, an early diagnosis.

In conclusion, there is a moderate to high likelihood that the population would agree to passive monitoring of cognition for the early detection of cognitive changes, depending on the sociocultural context of participants. Finally, it is of paramount importance that technologies ensure privacy and that participants understand the potential implications of early detection of cognitive disease prior to the use of a monitoring technology.

\section{Acknowledgement}

The authors would like to thank all participants, the UMass Center for Clinical and Translational Science, Dr. Susanne Muehlschlegel for assistance with the study design, and Dr. Sharina D. Person and Julie Flahive for assistance with statistical analysis.

\section{References}

1 Collaborators GB; GBD 2016 Dementia Collaborators. Global, regional, and national burden of Alzheimer's disease and other dementias, 1990-2016: a systematic analysis for the Global Burden of Disease Study 2016. Lancet Neurol. 2019 Jan;18(1):88-106.

2 Blennow K, Zetterberg H. The application of cerebrospinal fluid biomarkers in early diagnosis of Alzheimer disease. Med Clin North Am. 2013 May;97(3):369-76.

3 Slavin MJ, Brodaty H, Sachdev PS. Challenges of diagnosing dementia in the oldest old population. J Gerontol A Biol Sci Med Sci. 2013 Sep;68(9):1103-11.

4 Jack CR Jr, Bennett DA, Blennow K, Carrillo MC, Feldman HH, Frisoni GB, et al. A/T/N: An unbiased descriptive classification scheme for Alzheimer disease biomarkers. Neurology. 2016 Aug;87(5):539-47.

5 Babrak LM, Menetski J, Rebhan M, Nisato G, Zinggeler M, Brasier N, et al. Traditional and Digital Biomarkers: Two Worlds Apart? Digit Biomark. 2019 Aug;3(2):92-102.

\section{Statement of Ethics}

Research protocol (ID H00016303) was submitted to and approved by the University of Massachusetts Medical School Institutional Review Board. At the beginning of the survey, each participant provided written informed consent.

\section{Conflict of Interest Statement}

The authors have no conflicts of interest to declare.

\section{Funding Sources}

The authors received no funding for the research, authorship, and/or publication of this article.

\section{Author Contributions}

S.J.-H. took part in study design, literature review, data collection and analysis, data interpretation, and manuscript draft and revision. C.N. and J.H. took part in literature review and manuscript draft and revision. K.M.S. took part in study design, data analysis, and manuscript draft and revision.
6 Piau A, Wild K, Mattek N, Kaye J. Current State of Digital Biomarker Technologies for Real-Life, Home-Based Monitoring of Cognitive Function for Mild Cognitive Impairment to Mild Alzheimer Disease and Implications for Clinical Care: systematic Review. J Med Internet Res. 2019 Aug;21(8):e12785.

7 Kaye J, Mattek N, Dodge HH, Campbell I, Hayes T, Austin D, et al. Unobtrusive measurement of daily computer use to detect mild cognitive impairment. Alzheimers Dement. 2014 Jan;10(1):10-7.

8 Dodge HH, Mattek NC, Austin D, Hayes TL, Kaye JA. In-home walking speeds and variability trajectories associated with mild cognitive impairment. Neurology. 2012 Jun;78(24): 1946-52.

9 Austin J, Hollingshead K, Kaye J. Internet Searches and Their Relationship to Cognitive Function in Older Adults: Cross-Sectional Analysis. J Med Internet Res. 2017 Sep; 19(9):e307.

10 Minen MT, Gopal A, Sahyoun G, Stieglitz E, Torous J. The Functionality, Evidence, and Privacy Issues Around Smartphone Apps for the Top Neuropsychiatric Conditions. J Neuropsychiatry Clin Neurosci. 2020; DOI: 10.1176/appi.neuropsych.19120353.
11 Graham NL. Dysgraphia in dementia. Neurocase. 2000;6(5):365-76.

12 Owens DK, Davidson KW, Krist AH, Barry MJ, Cabana M, Caughey AB, et al.; US Preventive Services Task Force. Screening for Cognitive Impairment in Older Adults: US Preventive Services Task Force Recommendation Statement. JAMA. 2020 Feb;323(8): 757-63.

13 Vanderschaeghe G, Dierickx K, Vandenberghe R. Review of the Ethical Issues of a Biomarker-Based Diagnoses in the Early Stage of Alzheimer's Disease. J Bioeth Inq. 2018 Jun; 15(2):219-30.

14 Harris PA, Taylor R, Thielke R, Payne J, Gonzalez N, Conde JG. Research electronic data capture (REDCap)-a metadata-driven methodology and workflow process for providing translational research informatics support. J Biomed Inform. 2009 Apr;42(2):377-81.

15 Harris PA, Taylor R, Minor BL, Elliott V, Fernandez M, O’Neal L, et al.; REDCap Consortium. The REDCap consortium: building an international community of software platform partners. J Biomed Inform. 2019 Jul;95: 103208 . 
16 Aday LA, Cornelius LJ. Designing and conducting health surveys: a comprehensive guide. John Wiley \& Sons; 2006.

17 Kothare PA, Jadhav PR, Gupta P, Harrelson JC, Dickmann L. Harnessing the Potential of Emerging Digital Health and Biological Sampling Technologies for Clinical Drug Development: promise to Reality. Clin Pharmacol Ther. 2018 Dec;104(6):1125-35.
18 Martin S, Kelly S, Khan A, Cullum S, Dening T, Rait G, et al. Attitudes and preferences towards screening for dementia: a systematic review of the literature. BMC Geriatr. 2015 Jun;15(1):66.

19 Coravos A, Goldsack JC, Karlin DR, Nebeker C, Perakslis E, Zimmerman N, et al. Digital Medicine: A Primer on Measurement. Digit Biomark. 2019 May;3(2):31-71.
20 Mandryk RL, Birk MV. The Potential of Game-Based Digital Biomarkers for Modeling Mental Health. JMIR Ment Health. 2019 Apr;6(4):e13485.

21 Hsin H, Fromer M, Peterson B, Walter C, Fleck M, Campbell A, et al. Transforming Psychiatry into Data-Driven Medicine with Digital Measurement Tools. NPJ Digit Med. 2018 Aug;1(1):37. 